\title{
SYLLOGISMS WITH FRACTIONAL QUANTIFIERS
}

\begin{abstract}
Aristotle's syllogistic is extended to include denumerably many quantifiers such as 'more than $2 / 3$ ' and 'exactly $2 / 3$.' Syntactic and semantic decision procedures determine the validity, or invalidity, of syllogisms with any finite number of premises. One of the syntactic procedures uses a natural deduction account of deducibility, which is sound and complete. The semantics for the system is non-classical since sentences may be assigned a value other than true or false. Results about symmetric systems are given. And reasons are given for claiming that syllogistic validity is relevant validity.
\end{abstract}

\section{MOTIVATION}

The literature on syllogisms with fractional quantifiers such as 'at least $2 / 3$ ' contains no decision procedure for determining the validity of syllogisms with any finite number of premises. My main purpose is to give syntactic and semantic decision procedures for the validity of syllogisms, with any finite number of premises, where sentences contain the Aristotelian quantifiers - 'all,' 'no,' 'some,' and 'not all' - and the fractional quantifiers - 'more than $m / n$,' at least $m / n$,' 'exactly $m / n$,' 'less than $m / n$,' 'at most $m / n$,' and 'not exactly $m / n$.'

Geach ([5] pp. 61-64) considers syllogisms built from the fractional quantifiers 'more than $1 / 2$ ' and 'at least $1 / 2$.' He gives the following procedure, labelled a "decision procedure," for determining the validity of syllogisms with more than two premises: (1) Translate the premises and the denial of the conclusion into arithmetical claims about the cardinality of classes, (2) Use arithmetical reasoning to try to derive a contradiction, (3) If you derive a contradiction, label the argument valid, (4) If you do not derive a contradiction, look for a counterexample, and (5) If you find a counterexample, label the argument invalid. But this procedure is not a decision procedure in any strict sense, given its

Journal of Philosophical Logic 23: 401-422, 1994.

(C) 1994 Kluwer Academic Publishers. Printed in the Netherlands. 
references to arithmetical reasoning and to classes with unspecified cardinality.

For syllogisms with exactly two premises and with fractional quantifiers, decision procedures have been given. See Rescher and Gallagher [12] for a diagram decision procedure for two-premised syllogisms that may contain 'more than $1 / 2$,' 'at least $1 / 2$,' 'less than $1 / 2$,' and 'at most $1 / 2$.'

Below, "numerically definite" quantifiers such as ' 2 of the ... are among 3 of the ---' are not discussed. See De Morgan [3]. And, unlike Finch [4], we shall not assign true to any sentences of the form 'more than 2/3 of the A are B' if A denotes an infinite set. (But in the system below such a sentence may be true if $B$ denotes an infinite set.)

My main purpose is accomplished by proving Theorem 4 . The theorem is shown to generalize a result due to Smiley [13]. Theorem 5 in Section 5 generalizes a result due to Peterson ([11] §5.2) and another due to Meredith [8]. Section 6 describes general features of syllogistic validity. The final section summarizes the decision procedures that are discussed in the paper.

\section{PRELIMINARIES}

Syntax:

Terms: A, B, C, D, $\mathrm{A}_{1}, \mathrm{~B}_{1}, \ldots$

Aristotelian quantifiers: $A, E, I, O$

Relations: $>, \geq,=,<, \leq, \neq$

Fully reduced fractions greater than 0 and less than 1 (fractions):

$1 / 2,1 / 3,1 / 4,1 / 5 \ldots$

$2 / 3,2 / 5,2 / 7,2 / 9 \ldots$

$3 / 4,3 / 5,3 / 7,3 / 8 \ldots$

$\mathbf{P}_{m / n}$ is a fractional quantifier iff $\mathbf{P}$ is a relation and $m / n$ is a fraction.

(So, $>_{2 / 3}$ and $=_{4 / 5}$ are fractional quantifiers, but $>_{4 / 6}$ and $=_{5 / 4}$ are not. $\mathbf{P}$ is a quantifier iff $\mathbf{P}$ is an Aristotelian quantifier or a fractional quantifier. $\mathbf{P} a b$ is a sentence iff $\mathbf{P}$ is a quantifier and $a$ and $b$ are terms. $\mathbf{P} a b$ is a fractional sentence iff $\mathbf{P} a b$ is a sentence and $\mathbf{P}$ is a fractional quantifier. (Read $A \mathrm{AB}$ as 'All $\mathrm{A}$ are B,' $E \mathrm{AB}$ as 'No A are $\mathrm{B}$,' $I \mathrm{AB}$ as 'Some A are B,' $O A B$ as 'Some A are not B', $\mathbf{P}_{m / n} \mathrm{AB}$, where $\mathbf{P}$ is 
a fractional quantifier, as 'The ratio of A's that are B's to A's bears $\mathbf{P}$ to $m / n$.')

A non-empty finite set $\mathbf{X}$ of sentence is a chain iff the members of $\mathbf{X}$ can be arranged as a sequence of sentences such that any term that occurs in X occurs exactly twice and any term that occurs in a sentence in the sequence occurs in the sentence that succeeds it, where the first sentence in the sequence succeeds the last. (So, for example, $\left\{>_{1 / 3} \mathrm{AB}\right.$, $\left.E \mathrm{BC},<_{1 / 3} \mathrm{AC}\right\}$ is a chain. So is $\left\{<_{1 / 3} \mathrm{AA}\right\}$. But $\left\{<_{1 / 3} \mathrm{AB}\right\}$ is not.) $\langle\mathrm{X}, y\rangle$ is an $n$-premised syllogism iff $\mathrm{X}, y$ (that is, $\mathrm{X} \cup\{y\}$ ) is a chain, $\mathrm{X}$ is not a chain, and $\mathrm{X}$ has $n$ members $(n \geq 0)$. (So, $\langle\{A \mathrm{AB}\}, A \mathrm{AB}\rangle$ is a 1-premised syllogism, but $\langle\{A \mathrm{AA}\}, A \mathrm{AA}\rangle$ is not, since $\{A \mathrm{AA}\}$ is a chain.

$\langle\{A \mathrm{AB}, A \mathrm{BC}\}, A \mathrm{AC}\rangle$ is a 2-premised syllogism, but $\langle\{A \mathrm{AB}, A \mathrm{BA}\}, A \mathrm{AB}\rangle$ is not.) An $n$-premised syllogism is a syllogism. Evidence that Aristotle's focus was on syllogisms, as defined above, comes from Prior Analytics $42^{\mathrm{b}} 6-7$, where he says, when discussing valid arguments with two or more premises, that the number of terms exceeds the number of premises by exactly one.

$\langle\mathrm{U}, \mathfrak{J}\rangle$ is a model iff $\mathrm{U}$ is a non-empty set, $\mathfrak{I}$ is a function that assigns non-empty subsets of $\mathrm{U}$ to terms and assigns $t$ (true), $f$ (false), or $u$ (undetermined) to sentences, where the following conditions are met:

(1) $\mathfrak{I}(A a b)=t$ if $\mathfrak{I}(a) \subseteq \mathfrak{I}(b)$, and $f$, otherwise

(2) $\mathfrak{I}(E a b)=t$ if $\mathfrak{I}(a) \cap \mathfrak{I}(b)=\emptyset$, and $f$, otherwise

(3) $\mathfrak{I}(I a b)=t$ if $\mathfrak{I}(E a b)=f$, and $t$, otherwise

(4) $\mathfrak{I}(O a b)=t$ if $\mathfrak{I}(A a b)=f$, and $t$, otherwise

(5) $\mathfrak{I}\left(\mathbf{P}_{\alpha} a b\right)=u$ if $\mathfrak{I}(a)$ is infinite, $t$ if $\mathfrak{I}(a)$ is finite and $\overline{\overline{\mathfrak{I}(a) \cap \mathfrak{I}(b)}}+\overline{\overline{\mathfrak{I}(a)}} \mathbf{P} \alpha$, and $f$, otherwise

(So, for example, if $\langle U, \mathfrak{I}\rangle$ is a model where $U$ is the set of natural numbers, $\mathfrak{I}(\mathrm{A})$ is the set of natural numbers less than 10 and $\mathfrak{I}(B)$ is the set of even numbers, then $\mathfrak{I}\left(=_{4 / 9} \mathrm{AB}\right)=t$ and $\mathfrak{I}\left(=_{4 / 9} \mathrm{BA}\right)=u$.

By definition, a set $\mathrm{X}$ of sentences is (finitely) consistent iff there is a (finite) model $\langle\mathbf{U}, \mathfrak{J}\rangle$ such that $\mathfrak{I}$ assigns $t$ to each member of $\mathrm{X}$. A set of sentences is inconsistent iff it is not consistent. (So, for example,

$\left\{>_{2 / 5} \mathrm{AB},>_{2 / 5} \mathrm{AC}, E \mathrm{CB}\right\}$ is finitely consistent (and thus consistent), but $\left\{>_{2 / 5} \mathrm{AB},>_{3 / 5} \mathrm{AC}, E \mathrm{CB}\right\}$ is inconsistent.) By definition, $A a b^{*}={ }_{\mathrm{df}} O a b$, $E a b^{*}=_{\mathrm{df}} I a b, I a b^{*}=_{\mathrm{df}} E a b, O a b^{*}=_{\mathrm{df}} A a b,>_{\alpha} a b^{*}=_{\mathrm{df}} \leq_{\alpha} a b$, 
$\geq_{\alpha} a b^{*}==_{\mathrm{df}}<_{\alpha} a b,=_{\alpha} a b^{*}=_{\mathrm{df}} \neq_{\alpha} a b,<_{\alpha} a b^{*}=_{\mathrm{df}} \geq_{\alpha} a b$,

$\leq_{\alpha} a b^{*}=_{\mathrm{df}}>_{\alpha} a b$, and $\neq_{\alpha} a b^{*}==_{\mathrm{df}}=_{\alpha} a b$. (Read ' $. *^{*}=-\ldots$ ' as 'the contradictory of ... is - - .') Given a set $\mathrm{X}, y$ of sentences, $\langle\mathrm{X}, y\rangle$ is valid $\left(\mathrm{X} \models y\right.$ ) iff $\mathrm{X}, y^{*}$ is inconsistent. (So $\emptyset \models A \mathrm{AA}$ since $\{O \mathrm{AA}\}$ is inconsistent.) $\langle\mathrm{X}, y\rangle$ is invalid iff $\langle\mathrm{X}, y\rangle$ is not valid. $\langle\mathrm{X}, y\rangle$ is syllogistically valid $(\mathrm{X} \vDash s y$ ) iff $\langle\mathrm{X}, y\rangle$ is a syllogism and $\mathrm{X} \vDash y$. (So, for example, $A \mathrm{AB} \vDash s I \mathrm{AB}$ (keeping in mind that $\mathfrak{I}(\mathrm{A}) \neq \emptyset$ ). And $E \mathrm{AB} \vDash s \leq_{2 / 3} \mathrm{AB}$.)

Throughout the discussion lower-case letters, with or without subscripts, are used as metalinguistic variables that range over terms. $\mathbf{P}, \mathbf{Q}, \mathbf{P}_{\alpha}$, and $\mathbf{Q}_{\beta}$ are used as metalinguistic variables that range over quantifiers.

\section{NECESSARY CONDITION FOR SYLLOGISTIC VALIDITY}

Following Smiley [13], the expression $A a-b$ has this use: a set X of sentences has form $A a-b$ iff either $\mathrm{X}=\emptyset$ and $a=b$, or the members of $\mathrm{X}$ can be arranged as a sentence with the following form: $\left\langle A c_{1} c_{2}, \ldots A c_{i-1} c_{i}, A c_{i} c_{i+1}, \ldots A c_{n-1} c_{n}\right\rangle$ for $n>0$. So, for example, $\emptyset$ has form $A a-a$. $\{A \mathrm{AB}, A \mathrm{CD}, A \mathrm{BC}\}$ has form $A a-b$. (Make these replacements: $c_{1} / \mathrm{A}, c_{2} / \mathrm{B}, c_{3} / \mathrm{C}$, and $c_{4} / \mathrm{D}$.) But $\{A \mathrm{AB}, A \mathrm{BC}, A \mathrm{DC}\}$ does not have form $A a-b$. (The three members of the set cannot be arranged as a sequence in which the predicate of the first is the subject of the second and the predicate of the second is a subject of the third.)

THEOREM 1. X $\vDash s y$ only if $\mathrm{X}, y^{*}$ has one of the following $T$-forms:

(1) $A \mathrm{c}-\mathrm{a}, \mathbf{P c d}, A \mathrm{~d}-\mathrm{b}, E \mathrm{ab}$ (or $E \mathrm{ba}$ ), where $\mathbf{P}$ is $A, I,>_{\alpha}, \geq_{\alpha}$, or $=_{\alpha}$

(2) $\mathbf{P}_{\alpha} \mathrm{cd}, A \mathrm{~d}-\mathrm{a}, \mathbf{Q}_{\beta} \mathrm{ce}, A \mathrm{e}-\mathrm{b}, E \mathrm{ab}$, where

(i) $\mathbf{P}$ is $>$, $\geq$, or $=$ and $\mathbf{Q}$ is $>$, $\geq$, or $=$; and

(ii) If $>$ occurs then $\alpha+\beta \geq 1$, otherwise $\alpha+\beta>1$

(3) $A \mathrm{a}-\mathrm{b}, \mathbf{P a b}$, where $\mathbf{P}$ is $E, O,=_{\alpha},<_{\alpha}$, or $\leq_{\alpha}$

(4) $\mathbf{P}_{\alpha}$ ac, $A \mathrm{c}-\mathrm{b}, \mathbf{Q}_{\beta} \mathrm{ab}$, where

(i) $\mathbf{P}$ is $>$, $\geq$, or $=$ and $\mathbf{Q}$ is $<$, $\leq$, or $=$;

(ii) If $=$ does not occur twice then: $>$ or $<$ occurs and $\alpha \geq \beta$, or neither $>$ nor $<$ occurs and $\alpha>\beta$; and

(iii) If = occurs twice then: $\alpha>\beta$, or $\mathrm{c}=\mathrm{b}$ and $\alpha<\beta$

(5) $\mathbf{P}_{\alpha} \mathrm{ab}, \mathbf{Q}_{\alpha} \mathrm{ab}$, where both $=$ and $\neq$ occur 
( $\{A \mathrm{AB}, E \mathrm{AB}\}$ has T-form 1. Make these replacements: $\mathrm{a} / \mathrm{A}, \mathrm{b} / \mathrm{B}, \mathrm{c} / \mathrm{A}$, $\mathrm{d} / \mathrm{B}$, and $\mathbf{P} / A$. $\{E A \mathrm{~A}\}$ has $\mathrm{T}$-form 3. Make these replacements: a/A, $\mathrm{b} / \mathrm{A}$, and $\mathbf{P} / E$. Given the proof below, not $\left(\left\{\geq_{1 / 3} \mathrm{AB}, A \mathrm{BC}\right.\right.$, $\left.E C D\} \vDash<_{2 / 3} \mathrm{AD}\right)$ since $\left\{\geq_{1 / 3} \mathrm{AB}, A \mathrm{BC}, E \mathrm{CD}, \geq_{2 / 3} \mathrm{AD}\right\}$ does not have a $\mathrm{T}$-form. Note that the latter set could have no $\mathrm{T}$-form other than T-form 2, since this is the only T-form that involves $E$ and exactly two fractional quantifiers. This set cannot have T-form 2 given restriction (ii) for $\mathrm{T}$-form 2 . Note that $>$ does not occur and $\alpha+\beta=1$. In contrast, $\left\{\geq_{1 / 3} \mathrm{AB}, A \mathrm{BC}, E \mathrm{CD},>_{2 / 3} \mathrm{AD}\right\}$ has $\mathrm{T}$-form 2. Make these replacements: a/C, b/D, c/A, d/B, e/D, $\mathbf{P}_{\alpha} / \geq_{1 / 3}$, and $\mathbf{Q}_{\beta} />_{2 / 3}$.)

Proof. We use the following five lemmas.

LEMMA 1. If no fractional sentences occur in a chain $X$ that does not have a $T$-form then there is a model $\langle U, \mathfrak{J}\rangle$ such that $U$ has exactly three members and $\mathfrak{J}$ assigns $t$ to each member of $X$ (which means that $X$ is finitely consistent).

Proof. Use Johnson [6]. The result rests heavily upon Smiley [13].

LEMMA 2. If $A a_{1} a_{2}, W(X)$ and $E a_{1} a_{2}, W(Y)$ are finitely consistent, where $X$ and $Y$ are chains, $W \neq \emptyset$, and $\neq$ does not occur in $W$, then ${ }_{p / q} a_{1} a_{2}, W(Z)$ is finitely consistent.

Proof. Assume the antecedent. Let the distinct terms in $\mathrm{W}$ be $a_{1}, \ldots a_{n}(n \geq 2)$. Suppose $\left\langle\mathrm{U}_{1}, \mathfrak{I}_{1}\right\rangle$ and $\left\langle\mathrm{U}_{2}, \mathfrak{I}_{2}\right\rangle$ are finite models such that $\mathfrak{I}_{1}$ assigns $t$ to every member of $X$ and $\mathfrak{I}_{2}$ assigns $t$ to every member of $Y$. We give a three-staged construction of a model $\left\langle\mathrm{U}_{3}, \mathfrak{I}_{3}\right\rangle$ that shows that $\mathrm{Z}$ is finitely consistent. By definition, if $\langle\mathrm{U}, \mathfrak{I}\rangle$ is a model and $a_{1}, \ldots a_{n}$ are distinct terms then $\mathrm{C}_{1} \cap \ldots \mathrm{C}_{n}$ is an $a_{1}-a_{n}$ cell of $\mathfrak{I}$ iff $\mathrm{C}_{i}=\mathfrak{I}\left(a_{i}\right)$ or $\mathrm{C}_{i}=\overline{\mathfrak{I}\left(a_{i}\right)}$. (So there are $2^{n} a_{1}-a_{n}$ cells of $\mathfrak{J}$.)

Stage 1: construction of models $\left\langle\mathbf{U}_{1}^{\prime}, \mathfrak{I}_{1}^{\prime}\right\rangle$ and $\left\langle\mathbf{U}_{2}^{\prime}, \mathfrak{I}_{2}^{\prime}\right\rangle$. Suppose the $a_{1}-a_{n}$ cells of $\mathfrak{I}_{1}$ are $\mathrm{C}_{1}, \ldots \mathrm{C}_{2^{n}}$, the $a_{1}-a_{n}$ cells of $\mathfrak{I}_{2}$ are $\mathrm{D}_{1}, \ldots \mathrm{D}_{2^{n}}, \overline{\overline{\mathfrak{I}_{1}\left(a_{1}\right)}}=\alpha, \overline{\overline{\mathfrak{I}_{2}\left(a_{1}\right)}}=\beta$, and the least common multiple of $\alpha$ and $\beta$ is $\gamma$. Construct mutually disjoint sets $C_{1}^{\prime}, \ldots C_{2^{n}}^{\prime}$, $\mathrm{D}_{1}^{\prime}, \ldots \mathrm{D}_{2 n}^{\prime}$ such that $\overline{\overline{\mathrm{C}_{i}^{\prime}}}=\gamma / \alpha \times \overline{\overline{\mathrm{C}_{i}}}$ and $\overline{\overline{\mathrm{D}_{i}^{\prime}}}=\gamma / \beta \times \overline{\overline{\mathrm{D}_{i}}}$. Let $\mathrm{U}_{1}^{\prime}=\cup_{j=1}^{2^{n}} \mathrm{C}_{j}^{\prime}, \mathfrak{J}_{1}^{\prime}\left(a_{1}\right)=\cup_{j=1}^{2^{n}} \mathrm{C}_{j}^{\prime}$, where $\mathrm{C}_{j} \subseteq \mathfrak{I}_{1}\left(a_{i}\right)$, and for other 
terms $t$ let $\mathfrak{I}_{1}^{\prime}(t)=\mathbf{U}_{i}^{\prime}$. Construct $\left\langle\mathbf{U}_{2}^{\prime}, \mathfrak{I}_{2}^{\prime}\right\rangle$ in the same way (replacing ' $\mathrm{C}$ ' with ' $\mathrm{D}$ ', ' $\mathrm{U}_{1}$ ' with ' $\mathrm{U}_{2}$ ', and ' $\mathfrak{I}_{1}$ ' with ' $\mathfrak{J}_{2}$ '). So $\overline{\overline{\mathfrak{J}_{1}^{\prime}\left(a_{1}\right)}}=\overline{\overline{\mathfrak{J}_{2}^{\prime}\left(a_{1}\right)}}$.

Stage 2: construction of models $\left\langle\mathrm{U}_{1}^{\prime \prime}, \mathfrak{I}_{1}^{\prime \prime}\right\rangle$ and $\left\langle\mathrm{U}_{2}^{\prime \prime}, \mathfrak{I}_{2}^{\prime \prime}\right\rangle_{i \prime}$ Construct mutually disjoint sets $\mathrm{C}_{1}^{\prime \prime}, \ldots \mathrm{C}_{2^{\mathrm{n}}}^{\prime \prime}, \mathrm{D}_{1}^{\prime \prime}, \ldots \mathrm{D}_{2^{n}}^{\prime \prime}$ such that $\overline{\mathrm{C}}_{i}^{\prime \prime}=p \times \overline{\overline{\mathrm{C}}_{i}^{\prime}}$ and $\overline{\overline{\mathrm{D}_{i}^{\prime \prime}}}=q-p \times \overline{\overline{\mathrm{D}_{i}^{\prime}}}$. Let $\mathrm{U}_{1}^{\prime \prime}=\cup_{j=1}^{2^{n}} \mathrm{C}_{j}^{\prime \prime}, \mathfrak{J}_{1}^{\prime \prime}\left(a_{i}\right)=\cup_{j=1}^{2^{n}} \mathrm{C}_{j}^{\prime \prime}$, where $\mathrm{C}_{j}^{\prime} \subseteq \mathfrak{I}_{1}^{\prime}\left(a_{i}\right)$, and for other terms $t$ let $\mathfrak{I}_{1}^{\prime \prime}(t)=\mathrm{U}_{1}^{\prime \prime}$. Construct $\left\langle\mathrm{U}_{2}^{\prime \prime}, \mathfrak{I}_{2}^{\prime \prime}\right\rangle$ in a parallel manner (replacing ' $C$ ' with ' $D$ ', ' $U_{1}$ ' with ' $U_{2}$ ', and ' $\mathfrak{I}_{1}$ ' with ' $\mathfrak{I}_{2}$ ').

Stage 3: construction of model $\left\langle\mathrm{U}_{3}, \mathfrak{J}_{3}\right\rangle$. Let $\mathrm{U}_{3}=\mathrm{U}_{1}^{\prime \prime} \cup \mathrm{U}_{2}^{\prime \prime}$, and for any term $t, \mathfrak{J}_{3}(t)=\mathfrak{I}_{1}^{\prime \prime}(t) \cup \mathfrak{I}_{2}^{\prime \prime}(t)$.

The following example illustrates the above procedure. Consider the consistent sets $\left\{A \mathrm{AB},=_{1 / 2} \mathrm{BC},=_{2 / 3} \mathrm{AC}\right\}(\mathrm{X})$ and $\left\{E \mathrm{AB},=_{1 / 2} \mathrm{BC}\right.$, $\left.={ }_{2 / 3} \mathrm{AC}\right\}(\mathrm{Y})$. We follow the procedure and show the finite consistency of $\left\{={ }_{7 / 18} \mathrm{AB},=_{1 / 2} \mathrm{BC},=_{2 / 3} \mathrm{AC}\right\}(\mathrm{Z})$.

(1) The following diagrams indicate models that show the finite consistency of $\mathrm{X}$ and $\mathrm{Y}$ :
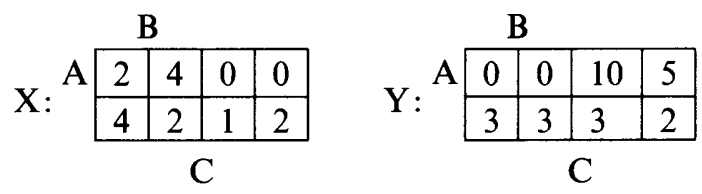

The number of members of cells is indicated. So, for example, in the model for X there are 6 B's that are C's and 12 B's. In the model for Y there are $3 \mathrm{~B}$ 's that are C's and $6 \mathrm{~B}$ 's.

(2) Given Stage 1, construct models:

B
$\mathrm{X}: \mathrm{A}$ \begin{tabular}{|l|l|l|l|}
\hline $2 \times 5$ & $4 \times 5$ & 0 & 0 \\
\hline $4 \times 5$ & $2 \times 5$ & $1 \times 5$ & $2 \times 5$ \\
\hline
\end{tabular}

$\mathrm{C}$

\begin{tabular}{|c|c|c|c|c|}
\hline \multicolumn{5}{|c|}{ B } \\
\hline & 0 & 0 & $10 \times 2$ & $5 \times 2$ \\
\hline & $3 \times 2$ & $3 \times 2$ & $3 \times 2$ & $2 \times 2$ \\
\hline
\end{tabular}


(No member of a cell in either model is a member of another cell in either model.)

(3) Given Stage 2, construct models:

$\mathrm{B}$
$\mathrm{X}: \mathrm{A}$
\begin{tabular}{|l|l|l|l|}
\hline $10 \times 7$ & $20 \times 7$ & 0 & 0 \\
\hline $20 \times 7$ & $10 \times 7$ & $5 \times 7$ & $10 \times 7$ \\
\hline
\end{tabular}

$\mathrm{C}$

B
$\mathrm{Y}: \mathrm{A}$
\begin{tabular}{|l|l|l|l|}
\hline 0 & 0 & $20 \times 11$ & $10 \times 11$ \\
\hline $6 \times 11$ & $6 \times 11$ & $6 \times 11$ & $4 \times 11$ \\
\hline
\end{tabular}

$\mathrm{C}$

(No member of a cell in either model is a member of another cell in either model.)

(4) Given Stage 3, construct model:

\begin{tabular}{|c|c|c|c|c|}
\hline \multirow{3}{*}{$\mathrm{Z}:{ }^{\mathrm{A}}$} & \multicolumn{2}{|c|}{ B } & & \\
\hline & 70 & 140 & 220 & 110 \\
\hline & 206 & 136 & 101 & 114 \\
\hline
\end{tabular}

We need to show that $=_{p / q} a_{1} a_{2}$ and any sentence in $\mathrm{W}$ is assigned $t$ by $\mathfrak{I}_{3}$. Given the procedure for constructing $\left\langle\mathrm{U}_{3}, \mathfrak{J}_{3}\right\rangle$,

$\overline{\overline{\mathfrak{I}_{3}\left(a_{i}\right) \cap \mathfrak{I}_{3}\left(a_{j}\right)}}=p \times \gamma / \alpha \times \overline{\overline{\mathfrak{I}_{1}\left(a_{i}\right) \cap \mathfrak{I}_{1}\left(a_{j}\right)}}+(q-p) \times \gamma / \beta \times$

$\overline{\overline{\mathfrak{I}_{2}\left(a_{i}\right) \cap \mathfrak{I}_{2}\left(a_{j}\right)}}$. And $\overline{\overline{\mathfrak{I}_{3}\left(a_{i}\right)}}=p \times \gamma / \alpha \times \overline{\overline{\mathfrak{I}_{1}\left(a_{i}\right)}}+(q-p) \times \gamma / \beta \times$

$\overline{\overline{\mathfrak{I}_{2}\left(a_{i}\right)}}$. Consider $={ }_{p / q} a_{1} a_{2} \cdot \overline{\overline{\mathfrak{I}_{1}\left(a_{1}\right) \cap \mathfrak{I}_{1}\left(a_{2}\right)}}=\overline{\overline{\mathfrak{I}_{1}\left(a_{1}\right)}}$,

$\overline{\overline{\mathfrak{I}_{2}\left(a_{1}\right) \cap \mathfrak{I}_{2}\left(a_{2}\right)}}=0$, and $\gamma / \alpha \times \overline{\overline{\mathfrak{I}_{1}\left(a_{1}\right)}}=\gamma / \beta \times \overline{\overline{\mathfrak{I}_{2}\left(a_{1}\right)}}$. So

$$
\frac{\overline{\overline{\mathfrak{I}_{3}\left(a_{1}\right) \cap \mathfrak{I}_{3}\left(a_{2}\right)}}}{\overline{\overline{\mathfrak{J}_{3}\left(a_{1}\right)}}}=\frac{p \times \frac{\gamma}{\alpha} \times \overline{\overline{\mathfrak{I}_{1}\left(a_{1}\right)}}}{q \times \frac{\gamma}{\alpha} \times \overline{\overline{\mathfrak{I}_{1}\left(a_{1}\right)}}}=\frac{p}{q} .
$$

So $\mathfrak{I}_{3}\left(={ }_{p / q} a_{1} a_{2}\right)=t$. Suppose $E a_{i} a_{j} \in \mathrm{W}$. Then $\overline{\overline{\mathfrak{I}_{1}\left(a_{i}\right) \cap \mathfrak{I}_{1}\left(a_{j}\right)}}=0$ and

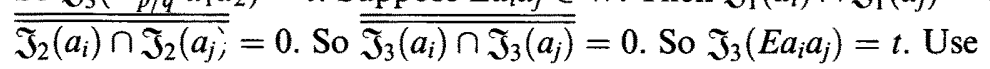


similar reasoning if $I a_{i} a_{j} \in \mathrm{W}$. Suppose $>_{\alpha} a_{i} a_{j} \in \mathrm{W}$. Then

$$
\begin{aligned}
& \frac{p \times \frac{\gamma}{\alpha} \times \overline{\overline{\mathfrak{I}_{1}\left(a_{i}\right) \cap \mathfrak{I}_{1}\left(a_{j}\right)}}}{p \times \frac{\gamma}{\alpha} \times \overline{\overline{\mathfrak{J}_{1}\left(a_{i}\right)}}}>\alpha \text { and } \\
& \frac{(q-p) \times \frac{\gamma}{\beta} \times \overline{\overline{\mathfrak{I}_{2}\left(a_{i}\right) \cap \mathfrak{J}_{2}\left(a_{j}\right)}}}{(q-p) \times \frac{\gamma}{\beta} \times \overline{\overline{\mathfrak{I}_{2}\left(a_{i}\right)}}}>\alpha .
\end{aligned}
$$

So

$$
\frac{\overline{\overline{\mathfrak{I}_{3}\left(a_{i}\right) \cap \mathfrak{I}_{3}\left(a_{j}\right)}}}{\overline{\overline{\mathfrak{I}_{3}\left(a_{i}\right)}}}>\alpha .
$$

(If $a / b>\alpha$ and $c / d>\alpha$ then $a+c / b+d>\alpha$.) So $\mathfrak{I}\left(>_{\alpha} a_{i} a_{j}\right)=t$. Use similar reasoning if $\mathbf{P}_{\alpha} a_{i} a_{j} \in \mathbf{W}$, where $\mathbf{P}$ is $\geq,=,<$, or $\leq$. Suppose $A a_{i} a_{j} \in \underline{\underline{\mathrm{W} . \overline{\mathfrak{I}_{3}\left(a_{i}\right) \cap \overline{\mathfrak{I}_{3}\left(a_{j}\right)}}}=p \times \gamma / \alpha \times \overline{\overline{\mathfrak{I}_{1}\left(a_{i}\right) \cap \overline{\mathfrak{I}_{1}\left(a_{j}\right)}}}+(q-p) \times}$

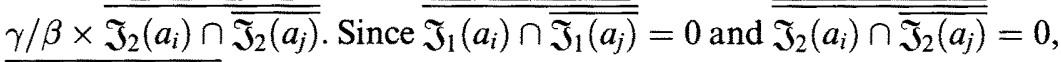
$\overline{\overline{\mathfrak{I}_{3}\left(a_{i}\right) \cap \overline{\mathfrak{I}_{3}\left(a_{j}\right)}}}=0$. So $\mathfrak{I}\left(A a_{i} a_{j}\right)=t$. Use similar reasoning if $O a_{i} a_{j} \in \mathrm{W}$. The following convention is used in the proofs of the next two lemmas: If $X$ is a set of sentences and $\mathbf{Q}$ is a fractional quantifier then $X_{Q . .\left.\right|_{A}}$ and $X_{Q}{ }_{\text {.. }}$ are sets of sentences formed by replacing $\mathbf{Q}$.. in X with $A$.. and $E$.., respectively. When there is no danger of ambiguity we shall shorten $\mathbf{Q}$.. to $\mathbf{Q}$. (If $\mathrm{X}$ is $\left\{>_{1 / 2} \mathrm{AB}, A \mathrm{BA}\right\}$ then $\mathrm{X}_{>1 / 2 \mid \mathrm{A}}$ is $\{A \mathrm{AB}, A \mathrm{BA}\}$ and $\mathrm{X}_{>_{1 / 2} \mid \mathrm{E}}$ is $\{E \mathrm{AB}, A \mathrm{BA}\}$. If $\mathrm{X}$ is $\left\{>_{1 / 2} \mathrm{AB},>_{1 / 2} \mathrm{BA}\right\}$ then $\mathrm{X}_{>\left._{1 / 2} \mathrm{AB}\right|_{\mathrm{A}}}$ is $\left\{A \mathrm{AB},>_{1 / 2} \mathrm{BA}\right\}$.)

LEMMA 3. If exactly one fractional sentence occurs in a chain $X$ that does not have a $T$-form then $X$ is finitely consistent.

Proof. Assume the antecedent. Suppose $\mathbf{Q}_{m / n}$. is the one fractional sentence in $\mathrm{X}$. There are four cases to consider.

Case 1: $\mathbf{Q}$ is $\neq$. If $\mathbf{X}_{\neq_{m / n} \mid \mathrm{A}}$ does not have a T-form, then $\mathrm{X}_{\neq_{m / n} \mid \mathrm{A}}$ is finitely consistent, by Lemma 1 . Then $\mathrm{X}$ is finitely consistent. (For, an interpretation that assigns $t$ to $A a b$ assigns $t$ to $\neq_{m / n} a b$.) If $\mathrm{X}_{f_{m / n} \mid \mathrm{A}}$ has a T-form, then X has form $A c-a, \neq_{m / n} c d, A d-b, E a b$ (or $E b a$ ). Then $\mathrm{X}_{\neq_{m / n} \mid \mathrm{E}}$ is finitely consistent, by Lemma 1 . Then $\mathrm{X}$ is finitely 
consistent. (For, an interpretation that assigns $t$ to $E a b$ assigns $t$ to $\neq_{m / n} a b$.)

Case 2. $\mathbf{Q}$ is $>$ or $\geq$. If $\mathbf{X}_{\mathbf{Q}_{m / n} \mid \mathrm{A}}$ does not have a $\mathrm{T}$-form then $\mathrm{X}$ is finitely consistent, by Lemma 1. Suppose $\mathrm{X}_{\mathbf{Q}_{m / n} \mid \mathrm{A}}$ has a $\mathrm{T}$-form. Then $\mathrm{X}$ has one of these forms:

Form 1.1. $A c-e,\left\{\begin{array}{l}>_{m / n} \\ \geq_{m / n}\end{array}\right.$ ef, $A f-a, A c d, A d-b, E a b$ (or $E b a$ ), where $c \neq e$.

Form 1.2. $A c-e,\left\{\begin{array}{l}>_{m / n} \\ \geq_{m / n}\end{array}\right.$ ef, $A f-a, I c d, A d-b, E a b$ (or $\left.E b a\right)$.

Form 1.3. $A c-a,\left\{\begin{array}{l}A \\ I \\ I\end{array} d, A d-e,\left\{\begin{array}{l}>_{m / n} \\ \geq_{m / n}\end{array} e f, A f-b, E a b\right.\right.$ (or Eba).

Form 1.4. $A a-c,\left\{\begin{array}{l}>_{m / n} \\ \geq_{m / n}\end{array} d, A d-b,\left\{\begin{array}{l}E \\ O\end{array} a b\right.\right.$, where $a \neq c$.

(Form 1.1's restriction $(c \neq e)$ and Form 1.5's restriction $(a \neq c)$ are required since we have assumed that $X$ does not have a $T$-form.) To show that $\mathrm{X}$ is finitely consistent if $\mathrm{X}$ has Form 1.1-1.3 let $\mathrm{U}=(1, \ldots m+1, m+2, \ldots n+1)$. For Form 1.1 let $\mathfrak{I}(e)=\mathrm{U}$, $\mathfrak{I}(f)=\{1, \ldots m+1\}$, and for other terms $t$ let $\mathfrak{I}(t)=\mathfrak{I}(f)$ if $A f-t \subseteq \mathrm{X}$, otherwise let $\mathfrak{I}(t)=\{m+2, \ldots n+1\}$. For Form 1.2 let $\mathfrak{I}(c)=\mathbf{U}, \mathfrak{I}(f)=\{1, \ldots m+1\}$, and for other terms $t$ let $\mathfrak{I}(t)=\mathfrak{J}(c)$ if $A c-t \subseteq \mathrm{X}$ and $\mathfrak{I}(t)=\mathfrak{I}(f)$ if $A f-t \subseteq \mathrm{X}$, otherwise let $\mathfrak{I}(t)=(m+2, \ldots n+1\}$. For Form 1.3 let $\mathfrak{I}(d)=\mathrm{U}$, $\mathfrak{I}(f)=\{1, \ldots m+1\}$, and for other terms $t$ let $\mathfrak{I}(t)=\mathfrak{I}(d)$ if $A d-t \subseteq \mathrm{X}$ and $\mathfrak{I}(t)=\mathfrak{I}(f)$ if $A f-t \subseteq \mathrm{X}$, otherwise let $\mathfrak{I}(t)=$ $\{m+2, \ldots n+1\}$. If $\mathrm{X}$ has Form 1.4 then $\mathrm{X}$ is finitely consistent since Form 1.4 is a special case of Form 1.3.

Case 3: $\mathbf{Q}$ is $<$ or $\leq$. If $\mathrm{X}_{\mathbf{Q}_{m / n} \mid \mathrm{E}}$ does not have a $\mathrm{T}$-form then $\mathrm{X}$ is finitely consistent, by Lemma 1 . If $X_{Q_{m / n} \mid E}$ has a $T$-form then $X$ has 
one of these forms:

Form 1.5. $A c-a, A c d, A d-b,\left\{\begin{array}{l}<_{m / n} \\ \leq_{m / n}\end{array} a b\right.$, where $c \neq a$.

Form 1.6. $A c-a, I c d, A d-b,\left\{\begin{array}{l}<_{m / n} a b . \\ \leq_{m / n}\end{array}\right.$

Form 1.7. $A c-a,\left\{\begin{array}{l}A \\ I\end{array} d, A d-b,\left\{\begin{array}{l}<_{m / n} \\ \leq_{m / n}\end{array} b a\right.\right.$.

To show that $\mathrm{X}$ is finitely consistent let $\mathrm{U}=\{1, \ldots m, \ldots n+1\}$. For Form 1.5 let $\mathfrak{I}(a)=\mathrm{U}$ and for other terms $t$ let $\mathfrak{I}(t)=\{1, \ldots m\}$. For Form 1.6 let $\mathfrak{I}(c)=\mathrm{U}$ and for other terms $t$ let $\mathfrak{I}(t)=\mathfrak{I}(c)$ if $A c-t \subseteq \mathrm{X}$, otherwise let $\mathfrak{I}(t)=\{1, \ldots m\}$. For Form 1.7 let $\mathfrak{I}(b)=\mathrm{U}$ and for other terms $t$ let $\mathfrak{I}(t)=\{1, \ldots m\}$.

Case 4. $\mathbf{Q}$ is $=$. If neither $\mathrm{X}_{=_{m / n} \mid \mathrm{A}}$ nor $\mathrm{X}_{=_{m / n} \mid \mathrm{E}}$ has a $\mathrm{T}$-form then $\mathrm{X}$ is finitely consistent, by Lemma 2 . If either $\mathrm{X}_{\bar{m} / n} \mid \mathrm{A}$ or $\mathrm{X}_{\bar{m}_{m / n} \mid \mathrm{E}}$ has a $\mathrm{T}$-form then $\mathrm{X}$ has one of Forms $1 . \mathrm{i} .1(1 \leq i \leq 7)$ constructed by replacing $\left\{\begin{array}{l}>_{m / n} \\ \geq_{m / n}\end{array}\right.$ in Forms $1.1-1.4$ and $\left\{\begin{array}{l}<_{m / n} \\ \leq_{m / n}\end{array}\right.$ in Forms 1.5-1.7 with $=_{m / n}$. Modify the models that show sets of Form 1.i are finitely consistent to show that sets of Form 1.i.1 are finitely consistent. (For example, suppose $\mathrm{X}$ has

Form 1.1.1. $A c-e,=_{m / n} e f, A f-a, A c d, A d-b, E a b$ (or $E b a$ ), where $c \neq e$.

Let $\mathrm{U}=\{1, \ldots m, m+1, \ldots n\}$. Let $\mathfrak{I}(e)=\mathrm{U}, \mathfrak{I}(f)=\{1, \ldots m\}$, and for other terms $t$ let $\mathfrak{I}(t)=\mathfrak{I}(f)$ if $A f-t \subseteq \mathrm{X}$, otherwise let $\mathfrak{I}(t)=\{m+1, \ldots n\}$.)

LEMMA 4. If exactly two fractional sentences occur in a chain $X$ that does not have a $T$-form then $X$ is finitely consistent.

Proof. Assume the antecedent. Suppose the two fractional sentences in $\mathrm{X}$ are $\mathbf{Q}_{m / n}$. and $\mathbf{R}_{p / q}--$. There are six cases to consider. 
Case 1: $\mathbf{Q}$ is $\neq$. Subcase $i$ : $\mathbf{R}$ is not $=$. Both $\mathrm{X}_{\mathbf{R}_{p / q^{--} \mid \mathrm{A}}}$ and $\mathrm{X}_{\mathbf{R}_{p / q-}-\mid \mathrm{E}}$ are finitely consistent, by Lemma 3 . So $\mathrm{X}$ is finitely consistent. Subcase $i i: \mathbf{R}$ is $=$. If either $\mathbf{X}_{f_{m / n} \mid \mathrm{A}}$ or $\mathrm{X}_{\neq_{m / n} \mid \mathrm{E}}$ have a $\mathrm{T}$-form. Then $\mathrm{X}$ is finitely consistent, by Lemma 3. Suppose both $\mathrm{X}_{\neq_{m / n} \mid \mathrm{A}}$ and $\mathrm{X}_{\neq_{m / n} \mid \mathrm{E}}$ have a $\mathrm{T}$-form. Then $\mathrm{X}$ has

Form 2.1. $A a-c, \neq_{m / n} c b,=p / q a b$, where $a \neq c$ or $m / n \neq p / q$.

Suppose $a \neq c$. Let $\mathrm{U}=\{1, \ldots p, \ldots q, \ldots r\}$, where $p / r \neq m / n$. And let $\mathfrak{J}(a)=\{1, \ldots q\}, \mathfrak{J}(b)=\{1, \ldots p\}$, and for other terms $t$ let $\mathfrak{I}(t)=\mathrm{U}$. Suppose $a=c$ and $m / n \neq p / q$. So $\mathrm{X}$ is $\neq_{m / n} a b,=_{p / q} a b$. Let $\mathrm{U}=\{1, \ldots p, \ldots q\}, \mathfrak{I}(a)=\mathrm{U}$, and for other terms $t$ let $\mathfrak{I}(t)=\{1, \ldots p\}$.

Case 2. $\mathbf{Q}$ is $>$ and $\mathbf{R}$ is not $\neq$. (Case 1 covers the situation in which $\mathbf{R}$ is $\neq$.) Subcase $i: \mathbf{R}$ is $>$ or $\geq$. If $\mathbf{X}_{\mathbf{Q}_{m / n} . \mid \mathrm{A}}$ does not have a $\mathrm{T}$-form then $\mathrm{X}$ is finitely consistent, by Lemma 3 . If $\mathrm{X}_{\mathbf{Q}_{m / n} . \mid \mathrm{A}}$ has a T-form then $\mathrm{X}$ has one of these forms:

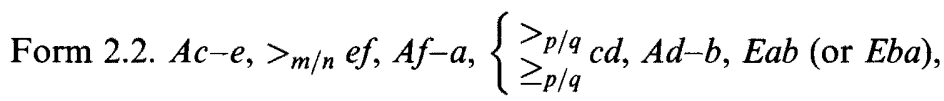
where $c \neq e$, or $m / n+p / q<1$.

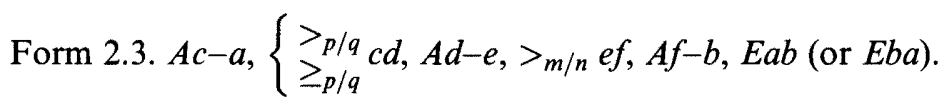

If $\mathrm{X}$ has Form 2.2, where $c \neq e$, or if $\mathrm{X}$ has Form 2.3, $\mathrm{X}$ is finitely consistent since $\mathrm{X}_{\mathbf{R}_{p / q}} c d_{\mid \mathrm{A}}$ is finitely consistent, by Lemma 3. Consider Form 2.2, where $c=e$ and $m / n+p / q<1$. Let $\mathrm{U}=\left\{1, \ldots m^{\prime}+1\right.$, $\left.m^{\prime}+2, \ldots r+1\right\}$, where $r$ is the least common denominator of $m / n$ and $p / q, m / n=m^{\prime} / r$, and $p / q=p^{\prime} / r$. (So $m^{\prime}+1 / r+1>m / n$. And $r-m^{\prime} /$ $r+1>p / q$. Let $\mathfrak{I}(c)=\mathrm{U}, \mathfrak{I}(f)=\left\{1, \ldots m^{\prime}+1\right\}$, and for other terms $t$ let $\mathfrak{I}(t)=\mathfrak{J}(f)$ if $A f-t \subseteq \mathrm{X}$, otherwise let $\mathfrak{I}(t)=\left\{m^{\prime}+2, \ldots r+1\right\}$. Subcase ii: $\mathbf{R}$ is $<$ or $\leq$. If $\mathrm{X}_{>_{m / n} \mid \mathrm{A}}$ does not have a $\mathrm{T}$-form then $\mathrm{X}$ is finitely consistent, by Lemma 3 . If $X_{>_{m / n} \mid A}$ has a $T$-form then $X$ has

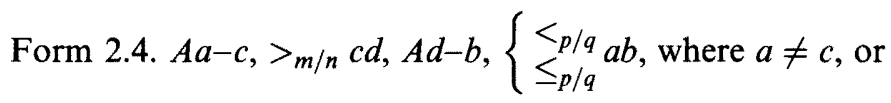
$m / n<p / q$. 
Suppose $a \neq c . \mathrm{X}_{\mathbf{R}_{p / q}} a b_{\mid \mathrm{E}}$ does not have a T-form. So $\mathrm{X}$ is finitely consistent, by Lemma 3. Suppose $a=c$ and $m / n<p / q$. Let $\mathrm{U}=\left\{1, \ldots m^{\prime}+1, \ldots r+1\right\}$, where $r$ is the least common denominator of $m / n$ and $p / q, m / n=m^{\prime} / r$, and $p / q=p^{\prime} / r$. (So $m^{\prime}+1 / r+1>m / n$. And $m^{\prime}+1 / r+1<p / q$.) Let $\mathfrak{I}(a)=\mathrm{U}$ and for other terms $t$ let $\mathfrak{I}(t)=\left\{1, \ldots m^{\prime}+1\right\}$. Subcase iii $: \mathbf{R}$ is $=$. If $\mathrm{X}_{>_{m / n} \mid \mathrm{A}}$ does not have a T-form then $\mathrm{X}$ is finitely consistent, by Lemma 3. If $X_{>_{m / n} ! A}$ has a $T$-form then $X$ has one of these forms:

Form 2.5. $A c-e,>_{m / n} e f, A f-a,={ }_{p / q} c d, A d-b, E a b$ (or $E b a$ ), where $c \neq e$, or $m / n+p / q<1$.

Form 2.6. $A c-a,{ }_{p / q} c d, A d-e,>_{m / n} e f, A f-b, E a b$ (or $E b a$ ).

Form 2.7. $A a-c,>_{m / n} c d, A d-b,=_{p / q} a b$, where $a \neq c$, or $m / n<p / q$.

To show that Forms $2.5,2.6$, and 2.7 are finitely consistent, modify the reasoning for Forms 2.2, 2.3, and 2.4, respectively.

Case 3: $\mathbf{Q}$ is $\geq$ and $\mathbf{R}$ is neither $\neq$ nor $>$. Use reasoning similar to that for Case 2.

Case 4: $\mathbf{Q}$ is $<$ and $\mathbf{R}$ is $=,<$, or $\leq$. Subcase $i: \mathbf{R}$ is $<$ or $\leq$. $\mathrm{X}_{\mathbf{Q}_{m / n} . \mid \mathrm{E}}$ is finitely consistent, by Lemma 3 . So $\mathrm{X}$ is finitely consistent. Subcase $i i: \mathbf{R}$ is $=. \mathrm{X}_{=p / q \mid \mathrm{E}}$ is finitely consistent, by Lemma 3. If $X_{=p / q \mid A}$ does not have a T-form then $X$ is finitely consistent, by Lemmas 3 and 2. If $\mathrm{X}_{=p / q \mid \mathrm{A}}$ has a T-form then $\mathrm{X}$ has

Form 2.8. $A a-c,={ }_{p / q} c d, A d-b,<_{m / n} a b$, where $a \neq c$ or $p / q<m / n$.

To show $\mathrm{X}$ is finitely consistent use reasoning similar to that for Form 2.4.

Case 5: $\mathbf{Q}$ is $\leq$ and $\mathbf{R}$ is $=$ or $\leq$. Use reasoning similar to that for Case 4.

Case 6: $\mathbf{Q}$ is $=$ and $\mathbf{R}$ is $=$. If neither $\mathrm{X}_{\mathbf{Q}_{m / n} . \mid \mathrm{A}}$ nor $\mathrm{X}_{\mathbf{Q}_{m / n} \mid \mathrm{E}}$ has a T-form then $\mathrm{X}$ is finitely consistent, by Lemma 2 . If $\mathbf{X}_{\mathbf{Q}_{m / n} . \mid A}$ has a T-form then $\mathrm{X}$ 
has one of these forms:

Form 2.9. $A c-e,=_{m / n} e f, A f-a,={ }_{p / q} c d, A d-b, E a b$ (or $E b a$ ), where $c \neq e$, or $m / n+p / q<1$.

Form 2.10. $A c-a,=_{p / q} c d, A d-e,=_{m / n} e f, A f-b, E a b$ (or Eba).

Form 2.11. $A a-c,=_{m / n} c d, A d-b,={ }_{p / q} a b$, where $a \neq c, d \neq b$ and $m / n \leq p / q$, or $m / n=p / q$.

Use reasoning similar to that for Forms $2.2,2.3$, and 2.4, respectively, to show that $\mathrm{X}$ is finitely consistent. If $\mathrm{X}_{\mathbf{Q}_{m / n} \mid \mathrm{E}}$ has a $\mathrm{T}$-form then $\mathrm{X}$ has one of these forms:

Form 2.12. $A c-a,=_{p / q} c d, A d-b,==_{m / n} a b$, where $c \neq a, d \neq b$ and $p / q \leq m / n$, or $p / q=m / m$.

Form 2.13. $A c-a,{ }_{p / q} c d, A d-b,={ }_{m / n} b a$.

Consider Form 2.12. Suppose $c \neq a$. Let $\mathrm{U}=\{1, \ldots p, \ldots q, q+1, \ldots r$, $\left.r+1, \ldots n^{\prime}\right\}$, where $m / n=m^{\prime} / n^{\prime}, m^{\prime}>p, n^{\prime}>q$, and $r+p-q=m^{\prime}$. Let $\mathfrak{I}(a)=\mathrm{U}, \mathfrak{I}(c)=\{1, \ldots q\}$, and for other terms $t$ let $\mathfrak{I}(t)=$ $\{1, \ldots p\} \cup\{q+1, \ldots r\}$. If $c=a$, then use the reasoning for Form 2.11. Consider Form 2.13. Let $\mathrm{U}=\{1, \ldots r, \ldots r / p \times q$, $(r / p \times q)+1, \ldots(r / p \times(q-p))+(r / m \times n)\}$, where $r$ is the least common multiple of $p$ and $m$. Let $\mathfrak{I}(c)=\{1, \ldots r / p \times q\}$ and for other terms $t$ let $\mathfrak{I}(t)=\mathfrak{J}(c)$ if $A c-t \subseteq \mathrm{X}$, otherwise let $\mathfrak{I}(t)=\{1, \ldots r\} \cup\{(r / p \times q)+1, \ldots(r / p \times(q-p))+(r / m \times n)\}$.

LEMMA 5. If more than two fractional sentences occur in a chain $X$ then $X$ is finitely consistent.

Proof. Assume the antecendent. Then at least two fractional sentences, $\mathbf{P} a b$ and $\mathbf{Q} c d$, occur in $\mathbf{X}$, where $a \neq c$. Let $\mathrm{Y}=\mathbf{X}-\{\mathbf{P} a b, \mathbf{Q} c d\}$. Construct sets $Y_{1}, \ldots Y_{n}$ by replacing every occurrence of $>_{\alpha}, \geq_{\alpha}$, or $=_{\alpha}$ in $\mathrm{Y}$ with $A$ and by replacing every occurrence of $=_{\alpha},<_{\alpha}, \leq_{\alpha}$, or $\neq_{\alpha}$ in $\mathrm{Y}$ with $E$. $\mathbf{Y}_{i} \cup\{\mathbf{P} a b, \mathbf{Q} c d\}$ is finitely consistent, by Lemma 4 . If $=$ does not occur then $n=1$, and thus $\mathrm{X}$ is finitely consistent. If $=$ occurs $m$ times $(m>0)$ then by $2^{m}-1$ uses of Lemma 2 it follows that $\mathrm{X}$ is finitely consistent. (So, for example, consider $X=\left\{>_{1 / 2} A B,=_{1 / 2} B C\right.$, $\left.>_{1 / 2} \mathrm{CD},=_{1 / 2} \mathrm{DA}\right\}$. Construct the following sets, which are finitely 
consistent, by Lemma 4: (1) $\left\{>_{1 / 2} \mathrm{AB}, A \mathrm{BC},>_{1 / 2} \mathrm{CD}, A \mathrm{DA}\right\}$, (2) $\left\{>_{1 / 2} \mathrm{AB}, A \mathrm{BC},>_{1 / 2} \mathrm{CD}, E \mathrm{DA}\right\},(3)\left\{>_{1 / 2} \mathrm{AB}, E \mathrm{BC},>_{1 / 2} \mathrm{CD}, A \mathrm{DA}\right\}$, and (4) $\left\{>_{1 / 2} \mathrm{AB}, E \mathrm{BC},>_{1 / 2} \mathrm{CD}, E \mathrm{DA}\right\}$. By Lemma $2,\left\{>_{1 / 2} \mathrm{AB}\right.$, $\left.A \mathrm{BC},>_{1 / 2} \mathrm{CD},=_{1 / 2} A \mathrm{DA}\right\}$ and $\left\{>_{1 / 2} \mathrm{AB}, E \mathrm{BC},>_{1 / 2} \mathrm{CD},=_{1 / 2} A \mathrm{DA}\right\}$ are finitely consistent. So, by Lemma $2, \mathrm{X}$ is finitely consistent.)

Theorem 1 is proved as follows. Suppose $\langle\mathbf{X}, y\rangle$ is a syllogism and $\mathrm{X}, y^{*}$ does not have a T-form. Then $\mathrm{X}, y^{*}$ is a chain. (For a term belongs to $y$ iff it belongs to $y^{*}$.) $\mathrm{X}, y^{*}$ is finitely consistent, given Lemmas $1,3,4$, and 5 . So not $(\mathrm{X} \vDash y)$. So $\mathrm{X} \vDash_{\mathrm{s}} y$ only if $\mathrm{X}, y^{*}$ has a T-form.

\section{SUFFICIENT CONDITION FOR SYLLOGISTIC VALIDITY}

We give a natural deduction (Aristotelian) account of deduction, using rules of inference, as in Smiley [13], instead of axioms, as in Lukasiewicz [7]. The rules of inference are:

(1) (Barbara) From $\mathbf{P} a b, A b c$ infer $\mathbf{P} a c\left(\mathbf{P}=A, I,>_{\alpha}\right.$, or $\left.\geq_{\alpha}\right)$.

(2) (Celarent) From $\mathbf{P} a b, E b c$ infer $\mathbf{Q} a c([\mathbf{P}, \mathbf{Q}]=[A, E],[I, O]$, $\left[>_{\alpha},<_{1-\alpha}\right]$, or $\left.\left[\geq_{\alpha}, \leq_{1-\alpha}\right]\right)$.

(3) (Baroco) From $\mathbf{P} a b, A c b$ infer $\mathbf{P} a c\left(\mathbf{P}=O,<_{\alpha}\right.$, or $\left.\leq_{\alpha}\right)$.

(4) (Felapton) From $\mathbf{P}_{\alpha} a b, \mathbf{Q}_{\beta} a c$ infer $O b c$ (Either $[\mathbf{P}, \mathbf{Q}]=[>, \leq]$ or $[\geq,<]$, where $\alpha \geq \beta$, or $[\mathbf{P}, \mathbf{Q}]=[\geq, \leq]$, where $\alpha>\beta)$.

(5) (E-conversion) from $E a b$ infer $E b a$.

(6) (Subordination) From $>_{\alpha} a b$ infer $\geq_{\alpha} a b$; from $=_{\alpha} a b$ infer $\geq_{\alpha} a b ;$ from $=_{\alpha} a b$ infer $\leq_{\alpha} a b ;$ and from $<_{\alpha} a b$ infer $\leq_{\alpha} a b$.

By definition, a sentence $y$ is deducible from a set $\mathrm{X}$ of sentences $(\mathrm{X} \vdash y)$ iff either $y \in \mathrm{X}$ or there is a sequence of sentences $\left\langle z_{1}, \ldots z_{n}\right\rangle$ such that (i) either $z_{k} \in \mathrm{X}, y^{*}$ or $z_{k}$ is entered by a rule of inference from previous members of the sequence, (ii) no rule of inference is allowed to introduce a sentence that has had a prior occurrence, and (iii) a sentence of form Eaa; Oaa, or $\leq_{\alpha} a a$ occurs in the sequence. (So, for example, $\left\{>_{1 / 2} \mathrm{AB}\right.$, $A \mathrm{BC}, E \mathrm{CD}\} \vdash<<_{1 / 2} \mathrm{AD}$, given the following sequence: (1) $>_{1 / 2} \mathrm{AB},(2)$ $A \mathrm{BC}$, (3) $E \mathrm{CD}$, (4) $\geq_{1 / 2} \mathrm{AD}$ (that is, $<_{1 / 2} \mathrm{AD}^{*}$ ), (5) $>_{1 / 2} \mathrm{AC}$ (from 1 and 2 by Barbara), (6) $<_{1 / 2} \mathrm{AD}$ (from 5 and 3 by Celarent), (7) $O \mathrm{DD}$ (from 4 
and 6 by Felapton). "Cesare," formed by replacing ' $E c b$ ' in Celarent with ' $E b c$ ', is among the derived rules of the system. Cesare is proven by using Celarent and E-conversion. "Darapti" (From Pab, Aac infer $I b c$, where $\mathbf{P}=A, I,>_{\alpha}$, or $\geq_{\alpha}$ ) is proven as follows. Suppose $\mathbf{P}=A$. Use this sequence: (1) $A a b$, (2) $A a c$, (3) $E b c$ (that is, $I b c^{*}$ ), (4) $E a c$ (from 1 and 3 by Celarent), (5) Eca (from 4 by E-conversion), (6) Eaa (from 2 and 5 by Celarent). Suppose $\mathbf{P}=I$ or $\geq_{\alpha}$. Use this sequence:

(1) $\mathbf{P} a b$, (2) $A a c$, (3) $E b c$, (4) $\mathbf{Q} a c$, where $Q=O$ or $\leq_{1-\alpha}$ (from 1 and 3 by Celarent), (5) $\mathbf{Q} a a$, where $\mathbf{Q}=O$ or $\leq_{1-\alpha}$ (from 4 and 2 by Baroco). Suppose $\mathbf{P}=>_{\alpha}$. Use this sequence: (1) $>_{\alpha} a b$, (2) $A a c$, (3) $E b c$, (4) $<_{1-\alpha} a c$ (from 1 and 3 by Celarent), (5) $<_{1-\alpha} a a$ (from 4 and 2 by Baroco), (6) $\leq_{1-\alpha} a a$ (from 5 by Subordination).)

\section{THEOREM 2. If $X \vdash y$ then $X \vDash y$.}

Proof. Assume the antecendent. There are two cases to consider.

Case 1: $y \in \mathrm{X}$. Then for any model $\langle\mathrm{U}, \mathfrak{I}\rangle$ such that $\mathfrak{J}$ assigns $t$ to each member of $\mathrm{X}, \mathfrak{J}$ assigns $t$ to $y$. So $\mathrm{X} \vDash y$.

Case 2: there is a sequence $\left\langle z_{1}, \ldots z_{n}\right\rangle$ as described in the definition of $\vdash$. First, each of the rules of inference passes truth downwards (that is, if $\langle\mathbf{U}, \mathfrak{I}\rangle$ is a model and $\mathfrak{I}$ assigns $t$ to the premises of a rule then $\mathfrak{I}$ assigns $t$ to the premises of a rule then $\mathfrak{I}$ assigns $t$ to the conclusion). (The reasoning is familiar if no fractional sentences occur in the premises of the rules. And there is no difficulty in showing that the Subordination rules pass truth downwards. Consider the other rules. Barbara, where $\mathbf{P}$ is $>_{\alpha}$. Suppose $\langle\mathbf{U}, \mathfrak{J}\rangle$ is a model, $\mathfrak{I}\left(>_{\alpha} a b\right)=t$, and $\mathfrak{I}(A b c)=t$. So $\mathfrak{I}(a)$ is finite and

$$
\frac{\overline{\overline{\mathfrak{I}(a) \cap \mathfrak{I}(b)}}}{\overline{\overline{\mathfrak{I}(a)}}}>\alpha
$$

Since $\mathfrak{I}(b) \subseteq \mathfrak{J}(c)$,

$$
\frac{\overline{\overline{\mathfrak{I}(a) \cap \mathfrak{I}(c)}}}{\overline{\overline{\mathfrak{I}(a)}}}>\alpha .
$$

So $\mathfrak{I}\left(>_{\alpha} a c\right)=t$. Use similar reasoning if $\mathbf{P}$ is $\geq_{\alpha}$. Celarent, where $\mathbf{P}$ is $>_{\alpha}$ and $\mathbf{Q}$ is $<_{1-\alpha}$. Suppose $\langle\mathbf{U}, \mathfrak{I}\rangle$ is a model, $\mathfrak{I}\left(>_{\alpha} a b\right)=t$, and 
$\mathfrak{J}(E b c)=t$. So $\mathfrak{I}(a)$ is finite and

$$
\frac{\overline{\overline{\mathfrak{I}(a) \cap \mathfrak{I}(b)}}}{\overline{\overline{\mathfrak{I}(a)}}}>\alpha \text {. }
$$

So

$$
\frac{\overline{\overline{\mathfrak{I}(a) \cap \overline{\mathfrak{I}(b)}}}}{\overline{\overline{\mathfrak{I}(a)}}}<1-\alpha .
$$

Since $\mathfrak{I}(c) \subseteq \overline{\mathfrak{I}(b)}$

$$
\frac{\overline{\overline{\mathfrak{I}(a) \cap \mathfrak{I}(c)}}}{\overline{\overline{\mathfrak{I}(a)}}}<1-\alpha
$$

So $\mathfrak{I}\left(<_{1-\alpha} a c\right)=t$. Use similar reasoning for the other values of $[\mathbf{P}, \mathbf{Q}]$. Baroco, where $\mathbf{P}$ is $<$. Suppose $\langle\mathrm{U}, \mathfrak{I}\rangle$ is a model, $\mathfrak{I}\left(<_{\alpha} a b\right)=t$, and $\mathfrak{I}(A c b)=t$. So, $\mathfrak{I}(a)$ is finite and

$$
\frac{\overline{\overline{\mathfrak{I}(a) \cap \mathfrak{I}(b)}}}{\overline{\overline{\mathfrak{I}(a)}}}<\alpha .
$$

Since $\mathfrak{I}(c) \subseteq \mathfrak{I}(b)$,

$$
\frac{\overline{\overline{\mathfrak{I}(a) \cap \mathfrak{I}(c)}}}{\overline{\overline{\mathfrak{I}(a)}}}<\alpha .
$$

So $\mathfrak{I}\left(<_{\alpha} a c\right)=t$. Use similar reasoning if $\mathbf{P}$ is $\leq_{\alpha}$. Felapton, where $\mathbf{P}$ is $>$ and $\mathbf{Q}$ is $\leq$. Suppose $\langle\mathrm{U}, \mathfrak{J}\rangle$ is a model, $\mathfrak{I}\left(>_{\alpha} a b\right)=t, \mathfrak{I}\left(\leq_{\beta} a c\right)=t$, and $a>\beta$. So, $\mathfrak{I}(a)$ is finite,

$$
\frac{\overline{\overline{\mathfrak{I}(a) \cap \mathfrak{I}(b)}}}{\overline{\overline{\mathfrak{I}(a)}}}>\alpha, \text { and } \frac{\overline{\overline{\mathfrak{I}(a) \cap \mathfrak{I}(c)}}}{\overline{\overline{\mathfrak{I}(a)}}} \leq \beta .
$$

So

$$
\frac{\overline{\overline{\mathfrak{I}(a) \cap \mathfrak{I}(c)}}}{\overline{\overline{\mathfrak{I}(a)}}}<\alpha .
$$


If $\mathfrak{I}(O b c)=f$ then

$$
\frac{\overline{\overline{\mathfrak{I}(a) \cap \mathfrak{I}(c)}}}{\overline{\overline{\mathfrak{I}(a)}}} \geq \frac{\overline{\overline{\mathfrak{I}(a) \cap \mathfrak{I}(b)}}}{\overline{\overline{\mathfrak{I}(a)}}}
$$

contradicting our previous assumptions. Use similar reasoning for the other values of $[\mathbf{P}, \mathbf{Q}]$.) Secondly, there is no model $\langle\mathbf{U}, \mathfrak{J}\rangle$ such that $\mathfrak{I}$ assigns $t$ to any of the following sentences: Eaa,Oaa, and $\leq_{\alpha} a a$. So, $\mathrm{X} \vDash y$.

THEOREM 3. If $X, y^{*}$ has a T-form then $X \vdash y$.

Proof. Assume the antecedent. For T-forms 1-4 we show how a sentence of form $\mathbf{Q} a a$, where $\mathbf{Q}$ is $E, O$, or $\leq_{\gamma}$, can be entered in a sequence that begins with the members of the T-form. For each T-form there are restrictions which must be noted. T-form 1: $A c-a$, $\mathbf{P} c d, A d-b, E a b$ (or $E b a$ ): Enter $\mathbf{P}^{\prime} c b$, where $\mathbf{P}^{\prime}$ is $A, I$, or $\geq_{\alpha}$, by Subordination (if necessary) and Barbara (if necessary); $E a b$, by $E$-conversion; $E c b$, by Barbara and Celarent (from $A c-a$ and $E a b$ ); $E b c$, by E-conversion; $\mathbf{Q} c c$, where $\mathbf{Q}$ is $E, O$, or $\leq_{1-\alpha}$, by Celarent (from $\mathbf{P}^{\prime} c b$ and $E b c$ ). T-form 2: $\mathbf{P}_{\alpha} c d, A d-a, \mathbf{Q}_{\beta} c e, A e-b, E a b$.

Suppose $\mathbf{P}$ is $>$. Enter $>_{\alpha} c a$, by Barbara; $\geq_{\beta} c e$, by Subordination; $\geq_{\beta} c b$, by Barbara; $E b a$, by E-conversion; $\leq_{1-\beta} c a$, by Celarent; $O a a$, by Felapton. Use similar reasoning if $\mathbf{P}$ is not $>$. T-form 3: $A a-b, \mathbf{P} a b$. If $a=b$, enter $\mathbf{P}^{\prime} a a$, where $\mathbf{P}^{\prime}$ is $E, O$, or $\leq_{\alpha}$, by Subordination. If $a \neq b$ enter $A a b$, by Barbara. If $\mathbf{P}$ is $E$ enter $E a a$ by E-conversion and Celarent. If $\mathbf{P}$ is not $E$ enter $\mathbf{Q} a a$, where $\mathbf{Q}$ is $O$ or $\leq_{\gamma}$, by Subordination and Baroco. T-form 4: $\mathbf{P}_{\alpha} a c, A c-b, \mathbf{Q}_{\beta} a b$. Suppose $\mathbf{P}$ is $>$. Enter $>_{\alpha} a b$, by Barbara; $\leq_{\beta} a b$, by Subordination: $O b b$, by Felapton. Use similar reasoning if $\mathbf{P}$ is not $>$. If $\mathbf{X}, y^{*}$ has T-form 5 , then $\mathrm{X}, y^{*}$ is $\left\{=_{\alpha} a b, \neq_{\alpha} a b\right\}$. Then $y \in \mathrm{X}$. Then $\mathrm{X} \vdash y$.

By definition, $\mathrm{X} \vdash_{\mathrm{T}} y$ iff $\langle\mathrm{X}, y\rangle$ is a syllogism and $\mathrm{X}, y^{*}$ has a T-form, and $\mathrm{X} \vdash_{\mathrm{S}} y$ iff $\langle\mathrm{X}, y\rangle$ is a syllogism and $\mathrm{X} \vdash y$.

THEOREM 4. $X \models_{S} y$ iff $X \vdash_{T} y$, and $X \models_{S} y$ iff $\mathrm{X} \vdash_{S} y$.

Proof. Suppose $\mathrm{X} \vdash_{\mathrm{s}} y$. So $\mathrm{X} \vdash_{\mathrm{T}} y$ (Theorem 1). So X $\vdash_{\mathrm{s}} y$

(Theorem 3). So $\mathrm{X} \vdash_{\mathrm{s}} y$ (Theorem 2). 
Aristotelian syllogisms are syllogisms that contain no fractional quantifiers.

COROLLARY 1 (Smiley [13]). If ' $X$, so $y$ ' is an Aristotelian syllogism then $X \models_{s} y$ iff $X, y^{*}$ has one of these forms, listed as they occur in Theorem 2 of ([13] p. 143): (i) $A a-b, O a b$, (ii) $A c-a, A c-b, E a b$, and (iii) $A c-a$, $A d-b, \operatorname{Icd}($ or $I d c), E a b$.

Proof. Form (i) has T-form 3 (where $\mathbf{P}=O$ ). Form (ii) has T-form 1. (If $\mathbf{P}=A$ then $\mathbf{P} c d, A d-b$ has form $A c-b$ ). Form (iii) has T-form 1 .

(If $I d c$, rather than $I c d$, occurs in form (iii) make these replacements in $A c-a, I c d, A d-b, E b a: a / b, b / a, c / d, d / c$.) $\mathrm{T}$-form 1 has form (ii) if $\mathbf{P}=A$ and form (iii) if $\mathbf{P}=I$. $\mathrm{T}$-form 3 has form (ii) if $\mathbf{P}=E$ and form (i) if $\mathbf{P}=O$. T-forms 2,4 , and 5 are irrelevant since their only instances are syllogisms in which there are fractional quantifiers.

\section{SYMMETRIC SYSTEMS}

We weaken the above language so that the only other quantifiers, if any, in addition to $A, E, I$, and $O$ are the finitely many fractional quantifiers $>_{\alpha_{1}}, \ldots>_{\alpha_{k}}, \leq_{\alpha_{1}}, \ldots \leq_{\alpha_{k}}$, where for each $>_{\alpha_{i}}$ there is an $>_{\alpha_{j}}$ such that $\alpha_{i}+\alpha_{j}=1$. We shall say that such a system is a symmetric system. (Note that $k$ is odd iff $>_{1 / 2}$ is one of the quantifiers in the system.)

Consider a symmetric system where the fractional quantifiers are $>_{3 / 4}$, $>_{1 / 2},>_{1 / 4}, \leq_{3 / 4}, \leq_{1 / 2}$, and $\leq_{1 / 4}$. Peterson ([9] pp. 355-356) points out that the 5-quantity syllogistic may, for some purposes, be expressed in this symmetric system. (For discussions of the 5 (and higher) - quantity syllogistic see [2], [9], [10], and [11].) Following Peterson, let the above six fractional quantifiers correspond to almost-all $(P)$, most $(T)$, many $(K)$, not-almost-all $(G)$, not-most $(D)$, and not-many $(B)$, respectively.

Peterson shows that there are exactly 105 valid two-premise syllogisms in this symmetric system. These 105 forms are listed in [9]. Among these forms are $A P T-1$ (' $A a b,>_{3 / 4} c a$; so $>_{1 / 2} c b$ '), $A D O-2$ (' $A a b, \leq_{1 / 2} c b$; so Oca'), PTI-3 ('> $>3 / 4 a b,>_{1 / 2} a c$; so $I c b$ '), and TAI-4 ('> $>_{1 / 2} a b, A b c$; so Ica'). $A P T-1$ is valid since ' $A a b,>_{3 / 4} c a, \leq_{1 / 2} a b$ ' has T-form 4. (Make these replacements: $a / c, b / b, c / a, \mathbf{P}_{\alpha} />_{3 / 4}$, and $\mathbf{Q}_{\beta} / \leq_{1 / 2}$.) $A D O-2$ is valid since ' $A a b, \leq_{1 / 2} c b, A c a$ ' has T-form 3. (' $A c_{1} c_{2}, A c_{2} c_{3}, \mathbf{P} c_{1} c_{3}$ ' is a 
special case of T-form 3. Make these replacements: $c_{1} / c, c_{2} / a, c_{3} / b$, and $\mathbf{P} / \leq_{1 / 2}$.) $P T I-3$ is valid since ' $>_{3 / 4} a b,>_{1 / 2} a c, E c b$ ' has T-form 2. (Make these replacements: $a / c, b / b, c / a, d / c, e / b, \mathbf{P}_{\alpha} />_{1 / 2}$, and $\mathbf{Q}_{\beta} />_{3 / 4}$.) $T A I-4$ is valid since ' $>>_{1 / 2} a b, A b c, E c a$ ' has T-form 1. (Make these replacements: $a / a, b / c, c / a, d / b, \mathbf{P} />_{1 / 2}$.)

Peterson ([9] p. 354) mentions his proof in ([11] §5.2) that for symmetric systems with $2 j$ quantifiers (including $A, E, I$, and $O$ ) there are exactly $3 j(j+2)$ valid syllogistic forms that have two premises. The following theorem generalizes this result.

THEOREM 5. For symmetric systems with exactly $2 k$ fractional quantifiers the number of valid $n$-premise syllogistic forms $(n \geq 2)$ is equal to $(n+1)\left(k+2+n\left(k^{2}+5 k+6\right) / 2\right)$.

Proof. For each T-form, taken in order, we give the number of forms of $n$-membered inconsistent sets $(n \geq 2)$ identified by it that are not identified by a preceding T-form. T-form 1: $2 n k+3 n+1$. (If $\mathbf{P}=A$, there are $n+1$ such forms. Note that $A c-a, A c-b, E a b$ and $A c-a, A c-b, E b a$ generate the same sets. If $\mathbf{P} \neq A$, there are $2 n(k+1)$ such forms.) T-form 2: $(n-1)\left(\sum_{i=1}^{k} i\right)$. (There are $n-1$ ways $\mathbf{P}$ and $\mathbf{Q}$ can be located in the set and there are $\left(\Sigma_{i-1}^{k} i\right)$ ways of meeting the restriction on T-form 2.) T-form 3: $(k+1)$. $(A a-b$, $E a b$ was identified as having T-form 1.) T-form 4: $\left(\Sigma_{i=1}^{k} i\right)$. T-form 5: 0 . So the number of forms of $n$-membered inconsistent sets $(n \geq 2)$ identified by T-forms is $(2 n k+3 n+1)+n\left(\sum_{i=1}^{k} i\right)+k+1$ (that is, $\left.k+2+n\left(k^{2}+5 k+6\right) / 2\right)$. Multiply this number by $n+1$ to find the number of valid syllogistic forms $\langle\mathrm{X}, y\rangle$, where $\mathrm{X}$ has $n$ members.

COROLLARY 1 (Peterson [11] §5.2). For symmetric systems with exactly $2 k$ fractional quantifiers the number of valid two-premise syllogistic forms is equal to $3 k^{2}+18 k+24$.

COROLLARY 2 (Meredith [8]). For symmetric systems with exactly 0 fractional quantifiers (that is, for Aristotelian syllogisms) the number of valid $n$-premise syllogistic forms $(n \geq 2)$ is equal to $3 n^{2}+5 n+2$. 


\section{RELEVANT VALIDITY}

Some "relevant" features of $=$ s are expressed in the following theorem.

THEOREM 6. (1) (Non-overlap) There are choices of $X$ and $y$ such that $y \in X$ and not $\left(X \models_{s} y\right)$. (2) (Non-dilution) There are choices of $X, y$ and $z$ such that $X \models_{s} y$ and not $\left(X, z \models_{S} y\right)$. (3) (Non-cut) There are choices of $X, y, z$ and $w$ such that $X \models_{s} y$, and $\{y, z\} \models_{s} w$, but not $\left(X, z \vDash_{S} w\right)$. (4) (Term sharing) If $X \vDash_{s} y$ and $X \neq \emptyset$ then any term that occurs in $y$ occurs in $X$. (5) (Non-trivial conclusion) If $X \models_{S} y$ and $X \neq \emptyset$ then there is a model $\langle U, \mathfrak{J}\rangle$ such that $\mathfrak{I}$ assigns $f$ to $y$. (6) (Premise consistency) If $X \models s y$ and $X \neq \emptyset$ then $X$ is consistent. (7) (Boethius's Thesis) IF $X \models_{s} y$ then not $\left(X \models_{S} y^{*}\right)$. (8) (Aristotle's Thesis) If $X, y \models_{S} z$ then not $\left(X, y^{*} \vDash_{S} z\right)$.

Proof. (1) Not $\left(\{A \mathrm{AB}, A \mathrm{BC}\} \models_{\mathrm{S}} A \mathrm{AB}\right)$. (2) $\left.\emptyset \models_{\mathrm{S}} A \mathrm{AA}\right\rangle$, but not $\left(\{A \mathrm{AB}\} \models_{\mathrm{S}} A \mathrm{AA}\right)$. (3) $\{A \mathrm{AB}, A \mathrm{BC}\} \models_{\mathrm{S}} A \mathrm{AC}$, and $\{A \mathrm{AC}, A \mathrm{CB}\} \models_{\mathrm{S}} A \mathrm{AB}$, but not $\left(\{A \mathrm{AB}, A \mathrm{BC}, A \mathrm{CB}\} \models_{\mathrm{s}} A \mathrm{AB}\right)$. (4) Use the fact that $\mathrm{X}, y$ is a chain. (5) Given the antecedent, two distinct terms occur in $y$. Models in which $y$ is false are easily constructed. (6) We use induction on the number $n$ of members of $\mathrm{X}$ to show there is a finite model that assigns $t$ to each member of X. Basis step: $n=1$. So $\mathbf{X}=\{\mathbf{P} a b\}$, where $a \neq b$. Finite models in which $\mathbf{P} a b$ is true are easily constructed. Induction step: $n>1$. By the induction hypothesis there is a model $\langle\{1, \ldots m\}, \mathfrak{J}\rangle$ such that $\mathfrak{J}$ assigns $t$ to each member of $(\mathbf{X}) \mathbf{P}_{1}\left(a b_{1}\right)$, $\mathbf{P}_{2}\left(b_{1} b_{2}\right), \ldots \mathbf{P}_{n}\left(b_{n-1} b_{n}\right)$, where the parentheses indicate that either term in a sentence may be the subject term. We use this model to construct a model that shows the consistency of $\mathrm{X}, \mathbf{P}_{n+1}\left(b_{n} c\right)$, where $c$ does not occur in $\mathrm{X}$.

Case 1: $\mathbf{P}_{n+1}$ is $A, I,>_{\alpha}, \geq_{\alpha}, \neq_{\alpha}$. Construct $\left\langle\mathbf{U}, \mathfrak{J}^{\prime}\right\rangle$, where $\mathfrak{I}^{\prime}(c)=\mathfrak{I}\left(b_{n}\right)$ and for other terms $t, \mathfrak{I}^{\prime}(t)=\mathfrak{I}(t)$.

Case 2: $\mathbf{P}_{n+1}$ is $E, O,<$, or $\leq$. Construct $\left\langle\mathrm{U}^{\prime}, \mathfrak{J}^{\prime}\right\rangle$, where $\mathrm{U}^{\prime}=\mathrm{U} \cup\{m+1\}, \mathfrak{J}^{\prime}(c)=\{m+1\}$ and for other terms $t \mathfrak{I}^{\prime}(t)=\mathfrak{I}(t)$.

Case 3: $\mathbf{P}_{n+1}\left(b_{n} c\right)$ is $={ }_{p / q} b_{n} c$. Construct $\left\langle\mathbf{U}^{\prime}, \mathfrak{J}^{\prime}\right\rangle$ where $\mathrm{U}^{\prime}=\{1 / 1, \ldots 1 / p, \ldots 1 / q\} \cup\{2 / 1, \ldots 2 / p, \ldots 2 / q\} \cup$ $\ldots\{m / 1, \ldots m / p, \ldots m / q\}$. For terms other than $c$ let $\mathfrak{I}^{\prime}(t)=\{x / i \mid 1 \leq i \leq q$ and $x \in \mathfrak{I}(t)\}$. Let $\mathfrak{J}^{\prime}(c)=\{x / i \mid 1 \leq i \leq p$ and $\left.x \in \mathfrak{I}\left(b_{n}\right)\right\}$. 
Case 4: $\mathbf{P}_{n+1}\left(b_{n} c\right)$ is $={ }_{p / q} c b_{n}$. Use a model similar to that for Case 3. (7) If X, $y^{*}$ has a T-form then $\mathrm{X}, y$ does not have a T-form.

(8) If $\mathrm{X}, y, z^{*}$ has a $\mathrm{T}$-form then $\mathrm{X}, y^{*}, z^{*}$ does not have a T-form.

Since both Aritotle's Thesis and Boethius's Thesis hold for $\vDash_{s}$, the above logic is a connexive logic, as defined by McCall ([1] p.435).

\section{DECISION PROCEDURES}

Given the above discussion there are three decision procedures for determining the validity of a syllogism $\langle\mathrm{X}, y\rangle$. The most practical is simply to determine whether $\mathrm{X}, y^{*}$ has a T-form. Another, also syntactic, decision procedure requires answering at most two questions: (1) Does $y \in X$ ? and (2) After listing all of the sentences in a deduction that begins with $\mathrm{X}, y^{*}$, does any sentence of the form Paa appear, where $\mathbf{P}$ is $E, O$, or $\leq_{\alpha}$ ? (Given the nature of the rules of inference at most finitely many distinct sentences can be entered, for no rule introduces a new term, and the number of new fractions that may be introduced is at most twice the number of fractions that occur in $\mathrm{X}, y$.) And this (impractical) semantic decision procedure is available. Use Theorem 4 and the reasoning for Theorem 1 to place an upper bound $n$ on the cardinality of the domains of models required to show the invalidity of $\langle\mathrm{X}, y\rangle$, if it is invalid. Check all models with domain $\{1, \ldots n\}$, determining whether any provides a counterexample, keeping in mind that any syllogism that can be shown invalid using a domain of size $m$ can be shown invalid using a domain of size $n$ if $n>m$. (So, for example, if $={ }_{4 / 5} \mathrm{AB}$ is the only fractional sentence that belongs to $\mathrm{X}, y$, then $(\mathrm{X} \vDash \mathrm{s} y)$ iff there is no counterexample in a model with domain $\{1, \ldots 15\}$.)

\section{ACKNOWLEDGEMENT}

I am grateful to an anonymous referee for several remarks that improved this paper. 


\section{REFERENCES}

[1] Anderson A. R. and N. D. Belnap, Jr., 1975, Entailment, vol. 1., Princeton, Princeton University Press.

[2] Carnes, R. D. and P. L. Peterson, 1991, Intermediate quantifiers versus percentages, Notre Dame Journal of Formal Logic 32, 294-306.

[3] De Morgan, A., 1847, Formal Logic, ed. by A. E. Taylor, London, Open Court, 1926.

[4] Finch, H. A., 1957, Validity rules for proportionally quantified syllogisms, Philosophy of Science 24, 1-18.

[5] Geach, P. T., 1976, Reason and Argument, Berkeley, University of California.

[6] Johnson, F., 1991, Three-membered domains for Aristotle's syllogistic, Studia Logica 50, 181-187.

[7] Lukasiewicz, J., 1957, Aristotle's Syllogistic, 2nd ed., Oxford, Clarendon Press.

[8] Meredith, C. A., 1953, The figures and models of the $n$-termed Aristotelian syllogisms, Dominican Studies 6, 42-47.

[9] Peterson, P. L., 1985, Higher quantity syllogisms, Notre Dame Journal of Formal Logic 26, 348-360.

[10] Peterson, P. L., 1991, Complexly fractionated syllogistic quantifiers, Journal of Philosophical Logic 20, 287-313.

[11] Peterson, P. L. and R. D. Carnes, The compleat syllogistic: Including rules and Venn diagrams for five quantities, MS, Syracuse University.

[12] Rescher, N. and N. A. Gallagher, 1965, Venn diagrams for plurative syllogisms, Philosophical Studies 26, 49-55.

[13] Smiley, T. J., 1973, What is a syllogism? Journal of Philosophical Logic 2, 136-154.

Department of Philosophy, Colorado State University,

Fort Collins,

CO 80523, U.S.A. 